\title{
Evaluation Research of Telecommunications Industry Knowledge Transfer Based on the Analytic Hierarchy Process
}

\author{
Gongyi Zhang \\ School of Management \\ Jilin University, Changchun, \\ China. \\ zgy516@163.com
}

\author{
Zhuoyi Wang \\ School of Management \\ Jilin University, Changchun, \\ China.
}

\author{
Lihong Sun \\ School of Management \\ Jilin University, Changchun, \\ China.
}

\begin{abstract}
To develop appropriate knowledge transfer strategy for China's telecommunications industry, the paper evaluates the status of the telecommunications industry knowledge transfer and establishes evaluation index system. Based on the AHP, the paper summarizes and analyzes the Telecommunications Industry knowledge transfer status, analysis shows that knowledge transfer capacity of the telecommunications industry is relatively higher, but the telecommunications industry knowledge transfer value network environment is still in its infancy. So the leaders of telecom industry value network -- telecom operators should strengthen the introduction of the knowledge transfer parties to create a better environment of the telecom industry knowledge transfer value network.
\end{abstract}

Key words-knowledge transfer; telecom industry; the analytic hierarchy process

I. The introduction of the Analytic Hierarchy Process and the Judgment Matrix Constructor

The Analytic Hierarchy Process (AHP) was put forward by a famous American operations research expert T.L.Saaty and other people in the 1970s. It is a combination of qualitative and quantitative analysis as well as multiple criteria decision making method. It is a decision-making method that the relevant elements of the decision problem is broken down into a hierarchy of objectives, guidelines, programs, and based on this to conduct qualitative and quantitative analysis. It makes the people's thinking process hierarchical, quantitative and uses the mathematics to provide quantitative basis in order to analyze, decide and forecast.

When using the AHP to analyze problem, we must put the problem hierarchical first. According to the nature of the problem and the total goal, the problem is decomposed into different elements, and we will combine these elements by different levels according to the interrelated factors and affiliation factors to form a multi-level analysis structure model. And finally the system analysis comes down to the lowest level (for the decision-making of the plan, measures, etc) relative to the highest level (total goal) of the determination of the relative importance of the weights or the order scheduling problem of the relative merits. In sorting calculation, the sort problem of each level factor relative to the previous level factors can be simplified to the judgment and comparison of the series pairs of factors. In order to make the comparative judgment quantitative, the AHP introduces a 1-9 scale method, and writes it into a form of judgment matrix. After the formation of the judgment matrix, then through the calculation of the biggest characteristic root and the corresponding characteristic, the relative importance of weights of a certain level factor relative to the previous level can be calculated. After calculating each factor's single-sort weight of a level relative to the previous level, using the weights of the previous level factor to weight composite can calculate the relative importance of weight of a level factor relative to the previous level. This is called the hierarchy total sort weights. In this way, the relative importance of weights or the ranking value of the relative merits order of the lowest level factors relative to the highest level can be calculated through top to bottom.

When the analysis hierarchical model has been built, then we can compare two factors in all factors, give the judgment of the relative importance of each level factor and show it with the appropriate scale. So the compare judgment matrix is constructed. The judgment matrix is not only the basic information of the AHP but also the important basis to calculate the relative importance.

Suppose that now we want to compare the influence that some factors $x=\left\{x_{1, \ldots,} x_{n}\right\}$ make to a certain factor $z$, Saaty etc suggested adopting the method that comparing between two factors to establish paired comparison matrix. It is can be interpreted that every time we take two factors $x_{i} 、 x_{j}$ and use $a_{i j}$ to express the influence of $z$ that $x_{i}$ and $x_{j}$ make. All comparison results can be expressed by matrix $A=\left(a_{i j}\right)_{n \times n}$, , $A$ is called pair-wise comparison matrix between $z-x$ ( the judgment matrix for short). Easy to see, if the ratio of the influence that $x_{i}$ make to $z$ and make $x_{j}$ to $z$ is $a_{i j}$ then the ratio between $x_{j}$ and $x_{i}$ make to $z$ should be $a_{j i}=1 / a_{i j}$

Definition 1 if matrix $A=\left(a_{i j}\right)_{n \times n}$, meet

$$
\text { ( i ) } a_{i j}>0, \quad \text { ( ii ) } a_{j i}=1 / a_{i j} i, j=1,2, \ldots n
$$

Then it is called the reciprocal matrix. (It is easy to see $\left.a_{i j}=1 \mathrm{i}, \mathrm{j}=1,2, \ldots n\right)$,

Concerning how to determine $\mathrm{a}_{\mathrm{ij}}$ Saaty etc suggested to quote numbers 1-9 and its countdown as the scale. Table 1 lists the meaning of the 1-9 scale: 
Table 1 the scale meaning of Analytic hierarchy process judgment matrix

\begin{tabular}{|l|l|l|}
\hline $\begin{array}{c}\text { Serial } \\
\text { number }\end{array}$ & The level of importance & \multicolumn{1}{|c|}{$a_{i j}$} \\
\hline 1 & $\begin{array}{l}\text { i,j the two factors are equally } \\
\text { important }\end{array}$ & 1 \\
\hline 2 & i is a little more important than $\mathrm{j}$ & 3 \\
\hline 3 & $\mathrm{i}$ is obviously more important than $\mathrm{j}$ & 5 \\
\hline 4 & $\mathrm{i}$ is strongly more important than $\mathrm{j}$ & 7 \\
\hline 5 & $\mathrm{i}$ is extremely more important than $\mathrm{j}$ & 9 \\
\hline 6 & $\mathrm{i}$ isn't a little more important than $\mathrm{j}$ & $1 / 3$ \\
\hline 7 & $\begin{array}{l}\mathrm{i} \text { isn't obviously more important } \\
\text { than } \mathrm{j}\end{array}$ & $1 / 5$ \\
\hline 8 & $\begin{array}{l}\mathrm{i} \text { isn't strongly more important than } \\
\mathrm{j}\end{array}$ & $1 / 7$ \\
\hline 9 & $\mathrm{i}$ isn't extremely more important & $1 / 9$ \\
\hline than $\mathrm{j}$
\end{tabular}

(Note: $a_{i j}=\{2,4,6,8,1 / 2,1 / 4,1 / 6,1 / 8\}$ means that the importance of grades rang among $\mathrm{a}_{\mathrm{ij}}=\{1,3,5,7,9$, $1 / 3,1 / 5,1 / 7,1 / 9\}$. These figures are determined based on the intuition and judgment that people deal with the qualitative analysis.

From the view of psychology, too much classification will go over people's judgment ability. In this way it not only increases the difficulty of judgment but also makes it easy to provide false data. Saaty etc also used experiment to compare the correctness of people's judgment in different scales. The result of the experiment also showed that using the 1-9 scale is the most appropriate.

\section{The principle of the building of telecom knowledge transfer status evaluation index system}

The design of the telecom industry knowledge transfer status evaluation index system is the basis to evaluate the telecom industry knowledge transfer result correctly and reasonably. The designation of the index system is essentially to establish a quantitative model of the evaluation in order to support the comprehensive evaluation of telecom industry knowledge transfer result and to provide data and decision support for the telecom industry who makes the decision of the knowledge transfer. Therefore, the evaluation index system should follow the following principles:

\section{A The Scientific Principles}

The scientific of the index system is the basis to ensure the evaluation results accurately and reasonably. Scientific principles ensure that the index system can reflect the basic characteristics of the telecom industry knowledge transfer effectively. Combined with the correct understanding of concept of the knowledge transfer effect factors, grasping the key factors of the telecom industry knowledge transfer status evaluation and ensuring the completeness of the evaluation index system and the logical rigor of the mathematical model make that the index system can scientific and effective reflect the telecom industry knowledge transfer result. It can also ensure the completeness and objectivity of the telecom industry knowledge transfer evaluation.

\section{B The Operability Principle}

Transferring knowledge in the economy system of telecom industry is the complicated system engineering. It is affected by the internal economy system and the external economy environment of the telecom industry. The performance of knowledge transfer depends not only on the investment of the knowledge transfer 、 the information level, the effort of knowledge transfer among different enterprises, but also on the consumption environment of the whole telecom industry and the influence and constraint of the advancement in telecom technology. Thus, we must consider the operability of the setting of the evaluation index and combine the qualitative and quantitative. From different dimensions we can reflect the status and the performance of the telecom industry knowledge transfer and ensure that the index is clear and the operation is easy.

\section{The Representative Principle}

The telecom industry knowledge transfer covers many levels in China. Each dimension of indexes has the situation of poor index independence such as the influence and compatible among each other. If all the indexes are included in the evaluation system, it is no doubt that it will evaluate more comprehensive of the status of telecom industry knowledge transfer. But this will increase the difficulty and workload of evaluation, and it will also lengthen the data acquisition cycle and affect the timeliness of the evaluation result. Therefore, when we design the index system, we will combine the reality of China's telecom industry and the demand of the knowledge transfer in telecom industry. We will select the representative indexes which could reflect the characteristics of telecom industry knowledge transfer among all dimensions to set up the evaluation index system.

\section{The establishment of the telecommunications industry}

knowledge transfer status evaluation index system

According to the requirements of the AHP, determining the evaluation system demands to define the target layer, rule layer and scheme layer of the ladder hierarchy.

In reference to a number of knowledge transfer evaluation, combining the interview results to the telecom experts and the senior leaders in telecom industry, we get the relevant indicators which can reflect the status and effect of the telecom industry knowledge transfer. Telecom industry knowledge transfer status evaluation system can be divided into seven levels. The telecom industry knowledge transfer status is evaluated as the overall objective (A). The assessment of knowledge transfer value network environment, the cognitive ability of knowledge transfer, the performance of knowledge transfer, the environment of knowledge transfer , the characteristics of knowledge transfer, the standards of the success of knowledge transfer etc seven aspects are evaluated as the second index ( the rule level $a_{i}$ ). At the same time, the indexes can be decomposed and refined based on the above dimensions. They can be refined into 24 indexes and they can construct an evaluation index target tree, shown in Figure 1.

\section{A As the basis of the status of knowledge transfer: the composite capacity indicators of the value network environment assessment (a1)}

1) The scale of the value network $\left(a_{11} / a_{31} / a_{41}\right)$

The scale of the value network is the most intuitive capacity indicators in the telecom enterprise value network environment and it affects the speed of the knowledge transfer in telecom enterprise value network. In general, when other factors are the same, the size of the value network is bigger the influence in the process of knowledge transfer will be greater but the overall speed of relative knowledge transfer will be slower. At the same time, the 
indicator is one of the key indexes which determine the cognitive ability of the value network knowledge transfer (a3) and the performance of the knowledge transfer (a4). From the present research results, the indicators and the two-tier criteria indicators are negative correlation.

2) The stability of the value network (a12/a32/a42)

The stability of the value network is the stability index of the telecom industry value network. Basing on the development trend of integration of the telecom industry, the cooperation among telecom enterprises becomes increasingly common, such as the strategic acquisitions, development of strategic cooperation and more and more open development cooperation. The overall compose a different telecom industry value network whose core is telecom operators. The stability of its network will determine this network's dominant extent of the process of knowledge transfer within other networks as well as the speed among enterprises in the same networks. At the same time, the indicator is one of the key indexes which determine the cognitive ability of the value network knowledge transfer (a3) and the performance of the knowledge transfer (a4). The indicators and the two-tier criteria indicators are positive correlation.

3) The dominate position of the operators in the value network (a13/a33/a44)

The operator's dominate position in the value network is the key indicator which can determine the speed of the response to the value network knowledge transfer

$B$ The speed indicators: the ability of knowledge transfer $\left(a_{2}\right)$

1) The ability of knowledge sender's knowledge transfer $\left(a_{21}\right)$.

As the knowledge sender's ability indicators, the ability of knowledge sender is that the sender explains the transfer's knowledge in appropriate ways within the organization and executes knowledge transfer. If the ability of sender's knowledge transfer is better, the knowledge transfer will be more successfully.

2) The knowledge transfer willingness of the knowledge sender $\left(a_{22}\right)$

The strength of the knowledge sender's transfer willingness will be directly related to the quantity and quality of the knowledge transferred. In general, the weeker the transfer willing of the knowledge transfer senders is, the more unwilling they will transfer the knowledge. So more and more knowledge will be deposited for this reason. In general, the stronger the willing of the knowledge transfer sender is, the more easily the transfer will succeed.

3) The ability of knowledge recipient's acceptance $\left(a_{23}\right)$

The ability of the knowledge recipient's acceptance is that recipients comprehend, absorb and make use of the value of the information.

4) The ability of knowledge recipient's transforming knowledge (a24)

The ability of knowledge recipient's transforming knowledge refers to the initiative that recipients take part in the knowledge transfer and the ability that recipients get knowledge from senders.
C The measurable indicators in the phase of the knowledge transfer: the cognitive ability of knowledge transfer (a3)

The cognitive ability of knowledge transfer is the indicators that measuring the phase of industry value network's knowledge transfer. Its strength relates to three sub-indicators (the size of the value network , the stability of the value network and the dominant position of operators in the value network) which introduced before.

\section{$D \quad$ The measurable indicators of the knowledge transfer result: the performance of the knowledge transfer (a4)}

The performance of knowledge transfer is the performance indicators that measure the industry value network knowledge transfer. Its strength relates to three sub-indicators (the size of the value network , the stability of the value network and the dominant position of operators in the value network) which introduced before.

\section{E The background indicators of knowledge transfer: the} environment of knowledge transfer (a5)

1) The geographical distance of knowledge transfer parties (a51)

The geographical distance of knowledge transfer parties will decide the extent of the difficulty, the requirement of time and the spending when knowledge transfer parties communicate face to face. The study found that in the process of knowledge transfer, the farther the knowledge transfer parties are away from each other, the speed of knowledge transfer is slower.

2) The knowledge gap among knowledge transfer parties (a52)

The knowledge gap among knowledge transfer parties refers that the knowledge's difference or similarity between knowledge senders and receivers. The knowledge gap between knowledge senders and receivers is smaller, the performance of the knowledge transfer is better.

3) The degree of cooperation and relationship among knowledge transfer parties (a53)

The degree of cooperation and relationship among knowledge transfer parties is the key factor that affects knowledge transfer. Droege \& Hoobler (2003) studied and showed that strong ties (refer that the relationship among acquaintances or people who close to each other) can inspire others to share their knowledge.

4) The system difference among knowledge transfer parties (a54)

The system difference among knowledge transfer parties is the difference of organization culture and value system of knowledge transfer parties. The more the same systems that knowledge transfer parties have, the performance of knowledge transfer is higher. Because knowledge transfer parties are not only able to anticipate and understand counterpart's working style, but also to adopt the similar approach to achieve.

\section{F The characteristics of the transferred knowledge (a6)}

The breakdown indicators of the characteristics of the transferred knowledge include tacit knowledge (a61) , knowledge embedded easily ( a62 ) and relationship embedded knowledge (a63) . The tacit knowledge is the codified and imparted easily knowledge. In general, tacit 
knowledge can be transferred easily. Knowledge embedded easily refers that the content transferred are embedded personnel or embedded tools. But relationship embedded knowledge need more communication and absorption among knowledge transfer parties. In short, because of the different characteristics of the knowledge transfer, the media of the knowledge transfer will be different. If knowledge can be transferred through coding or standardized transfer, knowledge transfer will be more easily.

\section{$G \quad$ The criteria of the success of knowledge transfer (a7)}

The criteria of the success of knowledge transfer include quantitative such as the acquisition of the necessary knowledge (a71) 、 enhance their own capabilities and the performance of business (a72). At the same time, the criteria of the success of knowledge transfer also include the breakdown indicators of stereotypes, such as the control of knowledge (a73) and the atmosphere of knowledge transfer

(a74) etc. In general, when the recipients of knowledge transfer obtain the knowledge their need, their own ability and the enterprise's performance will be promoted. That they obtain the control of the key knowledge and the trend of knowledge transfer is obvious, then you can think that knowledge transfer has obtained a certain success.

\section{The questionnaire of the telecom industry knowledge} transfer assessment and the result of the level analysis

After establishing the index system, we design a questionnaire according to the system. The questionnaire includes 《the weights questionnaire of telecom industry knowledge transfer situation assessment》 and 《the scoring questionnaire of telecom industry knowledge transfer situation assessment》. Through 《the scoring questionnaire of telecom industry knowledge transfer situation assessment》 we can acquire the scores that relevant persons give to each level of indications. Through 《the weights questionnaire of telecom industry knowledge transfer situation assessment》 we can get the index weights among the second level index a1,a2,a3...a7 and the weights among the third level index a11,a12,a13,..a $71, \mathrm{a} 72, \mathrm{a} 74$.

We have handed out 100 copies of questionnaire, 《the weights questionnaire of telecom industry knowledge transfer situation assessment 》 is 50copies and 《the scoring questionnaire of telecom industry knowledge transfer situation assessment》 is 50copies too. This questionnaire is mainly for telecom expert such as the senior managements of telecom industry, teachers of colleges and universities, the managements of telecom equipment supplier etc. We have got 43 copies of valid questionnaires after calculating the consistency proportional $\mathrm{CR}<0.1$.

\section{The determining of the telecom industry knowledge transfer evaluation index based on the AHP}

The measurement of each level index's evaluation weights shows the extent of importance of each level index. The weights and the size of influence that indexes make to telecom industry knowledge transfer is direct ratio, and the value is between $0-1$. The sum of weights of all statistical indicators factors equal to 1 . Among comprehensive assessment of the multi-factor, the correctness, rationality, scientific of weights affect that if the truth and objectivity of the final results can reflect the performance of the telecom industry knowledge transfer. Thus, we should be objective and cautious when we assign the weights of telecom industry knowledge transfer status index system. According to the general characteristics of assessment, we must combine the actual situation of telecom industry knowledge transfer to ensure the objective and effective of weights.

Since the source of the original data in calculation is different, the methods of determining the weights can be divided into subjective assignment method and objective assignment method. Subjective assignment is obtained mainly by experts who get the method by experience to judge subjectively such as the Delphi method, AHP, the paired factors comparison method etc. These methods are studied earlier and more mature, but the objectivity is poorer. The original data of the objective weighting method are formed from each index's actual data in assessment unit. It doesn't depend on the subjective judgment. So the objectivity of this kind method is stronger such as principal component analysis, maximizing deviation method, mean square deviation of the weight method and so on (Zhang yongyan,2007).

Because a number of experts in the survey set the weights of each level indexes, so we use the group analysis hierarchy process to deal with the weighted scores. The group analysis hierarchy process method in this paper is as follows: first, we use the data which is the corresponding location of the pairwise comparison matrix of the three indicators of the 43 experts to multiply scalar. Then, we use the MATLAB software to iterate the product to obtained 43 copies of geometric weighted average numbers. Through rounding these numbers, we obtain a judgment matrix of the next level index of the telecom industry knowledge transfer status evaluation $\mathrm{A}$, as shown in Table 2:

Table 2 the comparison between two matrixes of level A1 index of the measuring of telecom knowledge transfer status

\begin{tabular}{|l|l|l|l|l|l|l|l|}
\hline index & average & index & average & index & average & index & average \\
\hline $\mathrm{a} 11$ & 3.06 & $\mathrm{a} 24$ & 4.29 & $\mathrm{a} 43$ & 4.41 & $\mathrm{a} 62$ & 3.49 \\
\hline $\mathrm{a} 12$ & 2.78 & $\mathrm{a} 31$ & 3.56 & $\mathrm{a} 51$ & 4.49 & $\mathrm{a} 63$ & 4.67 \\
\hline $\mathrm{a} 13$ & 3.31 & $\mathrm{a} 32$ & 3.78 & $\mathrm{a} 52$ & 3.40 & $\mathrm{a} 71$ & 4.82 \\
\hline $\mathrm{a} 21$ & 3.33 & $\mathrm{a} 33$ & 3.91 & $\mathrm{a} 53$ & 2.33 & $\mathrm{a} 72$ & 3.57 \\
\hline $\mathrm{a} 22$ & 3.33 & $\mathrm{a} 41$ & 3.33 & $\mathrm{a} 54$ & 3.94 & $\mathrm{a} 73$ & 3.78 \\
\hline $\mathrm{a} 23$ & 4.11 & $\mathrm{a} 42$ & 3.33 & $\mathrm{a} 61$ & 3.87 & $\mathrm{a} 74$ & 3.39 \\
\hline
\end{tabular}

Through this we obtain the weights setting judgment matrix of the first level index. The largest eigenvalue of the judgment matrix is $\lambda \max =7.03$, the consistency check coefficient $\mathrm{CI}=0.02<0.1$. We will get the weight vector of first index of each level, $\mathrm{A}=(0.13,0.14,0.09,0.20,0.12$, $0.16,0.16$ ) after the corresponding eigenvector has been vector normalized.

Chart the system for measuring of telecommunications knowledge transfer status 


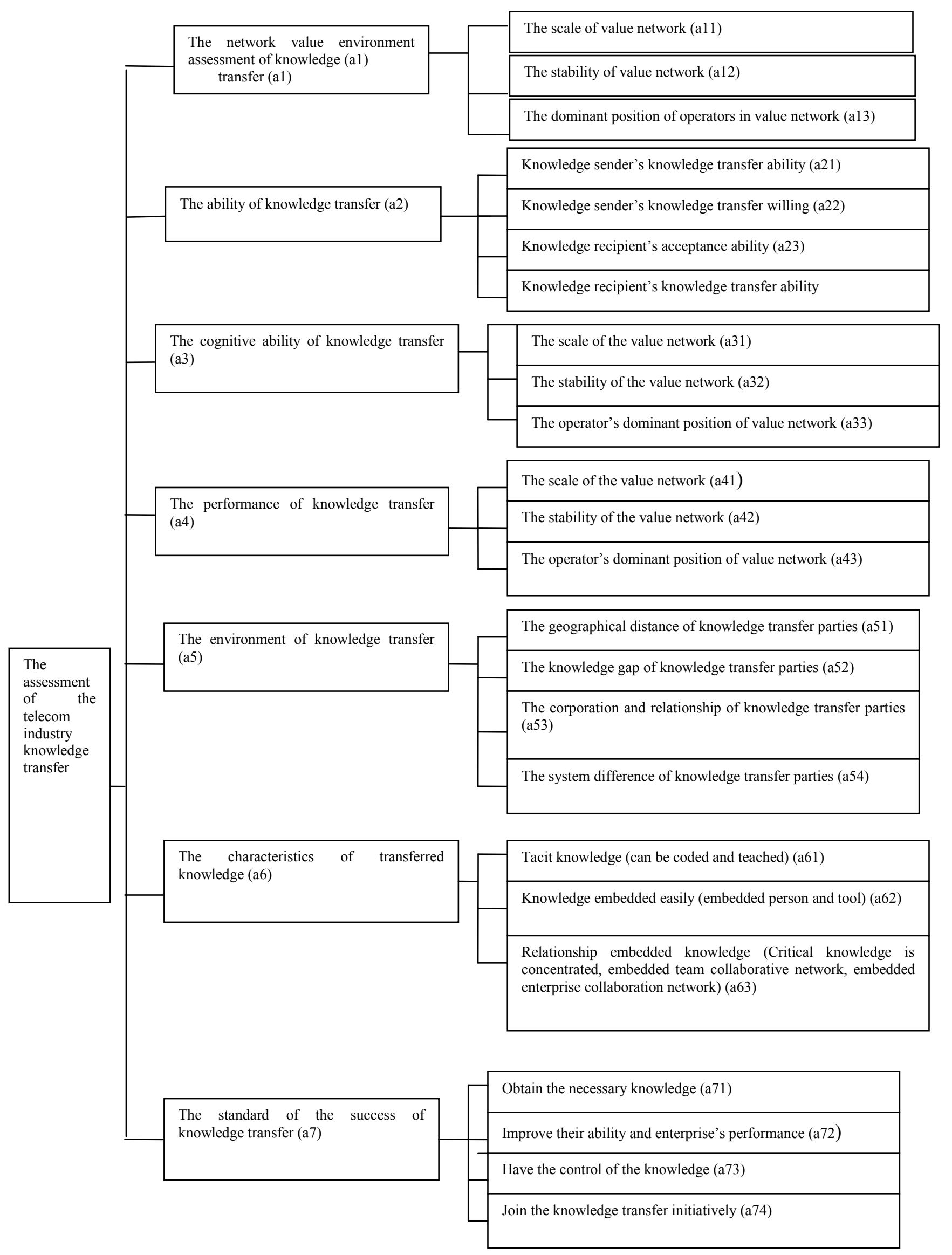


Similarly the weight of the second indicators can be obtained, $\mathrm{a} 1=(0.49,0.31,0.20) ; \mathrm{a} 2=(0.45,0.23,0.16,0.16)$; $\mathrm{a} 3=(0.14,0.53,0.33) ; \mathrm{a} 4=(0.14,0.43,0.43) ; \mathrm{a} 5=(0.13$, $0.07,0.25,0.55) ; \mathrm{a} 6=(0.21,0.35,0.44) ; \mathrm{a} 7=(0.41,0.14$, $0.22,0.22)$. The consistency of the calibration coefficient of the pairwise comparison matrix of the second index is less than 0.1 .

Using the above method we get the weight setting of the third indicators. We get the scores of the 43 copies second indicators by using the weights to weight the sores of the 43 copies third indicators. Then we calculate its average value to get the average scores of the second indicators. Then we use the weights of the second indicators which come from pairwise comparison matrix to weight the average value of second indicators. So we can get the scores of the first indicators. And strike the average value of that to get of the scores of the first indicators finally. The average value of the third level indicators are shown in Table 3

Table 3 the average scores of the third level indicators in the evaluation indicators system

\begin{tabular}{|c|c|c|c|c|c|c|c|}
\hline index & a1 & a2 & a3 & a4 & a5 & a6 & a7 \\
\hline a1 & 1 & 3 & 2 & 1 & 4 & 2 & $1 / 3$ \\
\hline a2 & $1 / 3$ & 1 & $1 / 2$ & 3 & 4 & $1 / 2$ & $1 / 3$ \\
\hline a3 & $1 / 2$ & 2 & 1 & $1 / 3$ & $1 / 5$ & 2 & 3 \\
\hline a4 & 1 & $1 / 3$ & 3 & 1 & $1 / 3$ & 2 & 4 \\
\hline a5 & $1 / 4$ & $1 / 4$ & 5 & 3 & 1 & $1 / 5$ & 3 \\
\hline a6 & $1 / 2$ & 2 & $1 / 2$ & $1 / 2$ & 5 & 1 & $1 / 2$ \\
\hline a7 & 3 & 3 & $1 / 3$ & $1 / 4$ & $1 / 3$ & 2 & 1 \\
\hline
\end{tabular}

Table 4 the average scores of the second level indicators in the evaluation indicators system

\begin{tabular}{|c|c|c|c|c|c|c|c|}
\hline index & a1 & a2 & a3 & a4 & a5 & a6 & a7 \\
\hline average & 3.02 & 3.61 & 3.79 & 3.79 & 3.57 & 4.19 & 4.05 \\
\hline
\end{tabular}

From table 4 we can see that the calculated average score of the second level indicators show that the assessment score of value network environment of knowledge transfer is 3.02 points; the assessment score of knowledge transfer ability is 3.28 points; the assessment score of knowledge transfer cognitive ability is 3.79 points; the assessment score of the performance of knowledge transfer is 3.79 points; the assessment score of the environment of knowledge transfer is 3.57 points; the assessment score of the characteristics of the transferred knowledge is 4.09 ; the assessment score of the standard status of knowledge transfer is 4.05 points.

The final score of the first level index of the telecom industry knowledge transfer status assessment A is 3.74 . From the scores we can know that the attention to the cognitive ability of knowledge transfer is the most currently. The investigating officers generally believe that the ability of telecom industry knowledge transfer has been relatively high. The characteristic of the transferred knowledge is conducive to the knowledge transfer of the telecom industry, but the value network environment of knowledge transfer is relatively in its infancy. Therefore, the telecom operators should strengthen the guidance of the value network members of knowledge transfer to create a better value network environment of the telecom industry knowledge transfer. In the aspect of cognitive ability of knowledge transfer, various constituents in the process of telecom industry knowledge transfer has a strong cognitive ability. They generally believe that the knowledge transfer of the telecom industry should be led by the telecom operators to strive for more partners to join the process of knowledge transfer. In this way the scale of knowledge transfer can be expanded and it can achieve win-win cooperation. When it comes that whether the knowledge transfer will succeed or not, it is generally believed that the knowledge transfer of the telecom industry will normally gain more success. In the aspect of the characteristics of the knowledge, the respondents believe that the transferred knowledge in the telecom industry is mostly the knowledge of relationship embedded. The critical knowledge is concentrated in certain enterprises to form a team of knowledge embedded or the collaborative network of knowledge transfer group. Knowledge diverts efficiently by embedding enterprise collaboration network. From the view of weights setting, the consistency of weights setting of the third level indexes of the experts is weaker. But from the view of the result of weights setting of the second indexes, most experts believe that in the process of the new telecom industry knowledge transfer, the weights of the consumers' rights indicators is the largest, followed by the indicators of optimizing the market structure, the weights of the indicators of supporting TD is the minimum. From the weight of the second level indictors as well as the score of the second level indictors and the final score, the reform of China's telecom industry has achieved good results.

\section{Relevant recommendations}

This paper evaluates the result of telecom industry knowledge transfer in China scientific by AHP. Firstly we introduce the AHP method, secondly we design the telecom industry knowledge transfer status evaluation index system, finally we evaluate the status of telecom industry knowledge transfer by using the AHP to obtain that all participating members of the telecom industry already have a good cognitive awareness of knowledge transfer and the ability of knowledge transfer. Knowledge transfer should be led by the telecom operators to create a harmonious environment for knowledge transfer, to strive for more partners to join the ranks of knowledge transfer, to form a win-win cooperation situation of knowledge transfer and accelerate the development of the telecom industry through knowledge transfer.

\section{Reference:}

[1] Zhang Yongyan. The fuzzy comprehensive evaluation of the network performance [D], the Master's thesis of Huazhong Normal University. 2007,P 21-24.

[2] Wang Feng, Liu Jingao, Chen Yahua. The research of the application of the computer system basing on the AHP telecom customer valuation model[J]. 2009.1:p26-p28

[3] Jiao Shufeng. The algorithm of the average random consistency index in AHP and the MATLAB implementation. The learned journal of Taiyuan Normal University (Natural Science) 2006.4. 